\title{
Dental Roots: Formation, Lengthening and Malformations of Roots
}

\author{
Michel Goldberg* \\ Department of Oral Biology, INSERM UMR-S1124 Paris Cite University, France
}

\begin{abstract}
The Hertwig's Epithelial Root Sheath (HERS) includes two layers: the Outer Enamel Epithelial Root Sheath and the Inner Enamel Epithelium Epithelium (IEE and OEE). They contribute both to the root formation. The inner columnar epithelial cells (IEE) of the dental papilla are formed by cells located near the dental papilla. They are at the origin of odontoblasts expressing FGF-4, -8 and -9 and at later stages BMP-2 and BMP7. The Outer Epithelial cells (OEE) express SHH, Msx2, enamel matrix proteins, paxillin, and Pax-6. When the cells of the HERS dissociate, intercellular spaces enlarge. Cells migrate from the epithelial sac, and underwent phenotypic inter conversion into cementoblasts and later cementocytes. The Hertwigs enamel epithelium contributes to cementum formation. Epithelial rest of Malassez is remnants of the Hertwig's root sheath. They are implicated in cementogenesis. Root lengthening and dentin thickening are involved in root elongation and dentin thickening to the detriment of the pulp chamber that is gradually reduced in volume. Apexogenesis and apexification contribute to root formation. Defects and abnormal root formation implicate missing teeth(hypodontia), orteeth in excess (supernumary teeth or hyperdontia) or mis-shapped structures. Genetic defects (Nfic, Ptc, Dkk1, Osx, Smad4, and $W l s$ ) have been identified. Premature arrests of root formation are due to apical infection, radiation, chemotherapy, as well as genes alterations. Roots malformations include root dilacerations which are abnormal curvature of the root and sharp bend of the crown or root axis. Taurodontism and other misshaped root structures are also frequently seen in man. Two main forms have been recognized: 1) the CLCN7 encoding a chloride channel, and 2) the second related to a defective PLG gene (encoding plasminogen). Altogether, theses defects contribute to major endodontic difficulties.
\end{abstract}

Keywords: Epithelial hertwig's root sheath, OEE, IEE, Cell rests of malassez, Apexogenesis apexification, Acellular cement, Cellular cement, Taurodontism, Root dilacerations, Dental type I and type III, Dysplasia, Hypophosphatasia

After the crown formation, the roots are lengthening and extending below the cervical loop, where the enamel organ fuse. The Epithelial Enamel Root Sheath (HERS) is reduced to a double layer. Instead of the four layers of the enamel organ. The outer enamel epithelium, and the inner enamel epithelium contribute efficiently to the root formation.

\section{Root Anatomy}

The root formation is under the control of the Hertwig's Epithelial Root Sheath (HERS). Mesenchymal cells of the dental papilla differentiate into odontoblasts. It is a bilayer originated from the apical region of the enamel organ. HERS is guiding root formation determining the size, shape and number of tooth roots. HERS is a proliferation of epithelial cells located at the cervical loop of the enamel organ. The epithelial root sheath consists of confluent outer and inner epithelial strata, in some cases enclosing a central layer similar to the stratum intermedium. C14 and PCNA are expressed in the HERS, as well as Insulin-like growth factor. In most cases, HERS is essentially formed by two layers: the outer and inner layers at the origin of roots(lengthening and thickening) and apex closure (apexogenesis and apexification).
Quick Response Code:

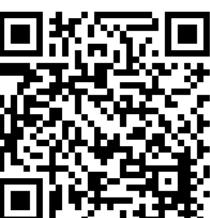

*Corresponding author: Michel Goldberg, Faculty of Fundamental and Biomedical Sciences, INSERM UMR-S1124 Paris Cite University, 45 rue des Saints Pères, Paris, France

Received: 05 October, 2021

Published: 28 October, 2021

Citation: Goldberg M. Dental Roots: Formation, Lengthening and Malformations of Roots. SOJ Den Oral Disor. 2021;1(3):1-10. DOI: 10.53902/SOJDOD.2021.01.000514 


\section{Inner \& Outer HERS}

\section{The Inner layer (or internal EE) are tall columnar cells located near the dental papilla of the enamel organ}

The cells are oval, with large nucleus and narrow rough endoplasmic reticulum (RER). They elongate and acquire a columnar shape. The number of RER cisternae increases. They are connected by gap junctions, desmosomes, hemi-desmosomes and immature tight junctions. The columnar cells of the inner enamel epithelium are at right angles to the basement membrane and they isolate the epithelial cells from the dental papilla.

The inner epithelial (IEE) cells gradually began to separate. HERS is guiding root formation determining the size, shape and number of tooth roots. The odontoblast processes establish contact with the basal lamina of sheath cells. There is a transient increase in RER. The IEE expressed FGFs-4, -8 , and -9 , BMP-4, and IGF1, and at later stage, BMP2 and BMP7. The IEE acquire the function in secreting the enamel matrix. The cells become tall, columnar and accumulate cell organelles. They are called preameloblats, and constitute the enamel forming cells. The mechanisms involved are a reciprocal induction. The epithelial sheath is a continuation of the outer and inner epithelium. Ki67-positive cells suggest cell proliferation and the OEE cells are dividing more actively than the IEE. The IEE differentiate into odontoblasts that secrete dentin, namely root dentin.

\section{Formation of the cervical loop and HERS}

After fragmentation and formation of the dentin follicle, the cells come in direct contact with the root dentin and differentiate into cementoblasts. Thereafter, the columnar cells become discontinuous. II was observed that the cells of the OEE broke before the IEE. When the cells begin to separate, some odontoblast processes made contact with the basal lamina with a transient increase in RER.

\section{The Outer Enamel Epithelium (OEE) (or external EE)}

The Outer Enamel Epithelium Is a layer expressing SHH, MSX2 and enamel matrix proteins? The outer layer is formed by cuboidal cells, three to four layers of cells, with intercellular open spaces. They are connected by desmosomes, gap junctions and they display a thin cytoplasmic border. Occasionally flat tight junctions are visible. A basement membrane separates the enamel epithelium from the surrounding connective tissue of the dental sac. Paxillin is observed with strong labeling in the OEE and in the apex of the cervical loop (CL). OEE cells are cuboidal. They contain more organelles than the IEE and exhibits strong Pax6 expression, and also SHH. The cervical loop is the location where the outer EE and the inner EE join. Bmp-2, Bmp-4 and Msx-2 are expressed in HERS during root formation. Laminin beta- 3 and syndecan 1 indicate that cell adhesion and proliferation are essential for HERS development. Amelin and amelogenin are located along the epithelial root sheath of fully formed rat molars. BMP-4 regulates the formation of HERS. Ameloblastin (AMBN) is not expressed during tooth development. Perlecan, and the removal of the heparan sulfate HSPG perlecan are important steps for root and periodontal tissue formation. Heparanase secreted by the cells of HERS contribute to root formation by degrading perlecan. Hirata \& Nakamura ${ }^{1}$ It critically depends on nuclear factor Ic (Nfic) and transforming factor beta (Tgf $\beta$ ) signaling, this later being mediated by Smad $4 .^{2}$

It is also possible that HERS undergo an epithelial-mesenchymal transformation (EMT) (as phenotypic inter conversion) and become functional cementoblasts. Sub-population of HERS has ameloblastin (amelin) and Dlx-2 as definite markers. They do not synthesize amelogenin or enamelin. They transcribe ALP, Shh (sonic hedgehog) and other proteins. In contrast, vimentin and N-cadherin act as mesenchymal markers. Snail-1 and -2, and TNALP-keratin are present and strongly expressed in HERS. CK14 and PCNA are transcribed in the HERS. Insulin-like growth factor is abundantly present in the outer layer of HERS.

One group of cells showed TUNEL reactivity. They are embedded in the cementum and thereafter die by apoptosis. The other group of cells survives in the cement and constitutes epithelial cell rests of Malassez, located in the alveolo-dentinal ligament and/or embedded in the cementum. A possible role of apoptosis and epithelium-mesenchymal transition (interconversion) are also associated during HERS maturation. A number of pathologies are associated with HERS, the key molecules for theses being Osterix; $\beta$ cathenin, and Shh. They are also combined with enamel pearls, periapical cyst, accessory root canals, and periodontal pocket. Tooth abnormalities are classified as dilacerations; extra-root formation, double root formation, concrescence, rizomegali and hypercementozis.

\section{Root furcations}

The critical structure for furcation is a tongue-shaped epithelial projection from the cervical loop of the enamel organ that remains inactive during crown formation of the bi- or tri-furcation. ${ }^{3}$ The tongue proliferates and unites to form a continuous bridge. These bridges induce the differentiation of odontoblasts which produce the dentin at the floor of the pulp cavity Figure 1.

The formation of bifurcation and trifurcation of roots Sicher. ${ }^{4}$ Epithelial projections taking origin in the cervical loop proliferate, and merge forming a continuous separation into two or three areas. Anterior teeth have a single root, while deciduous and permanent molars have two roots in the mandible and three roots in the maxilla. The formation of three roots takes place in the maxillary molars which possess three roots dividing the apical space Figure 2 . 


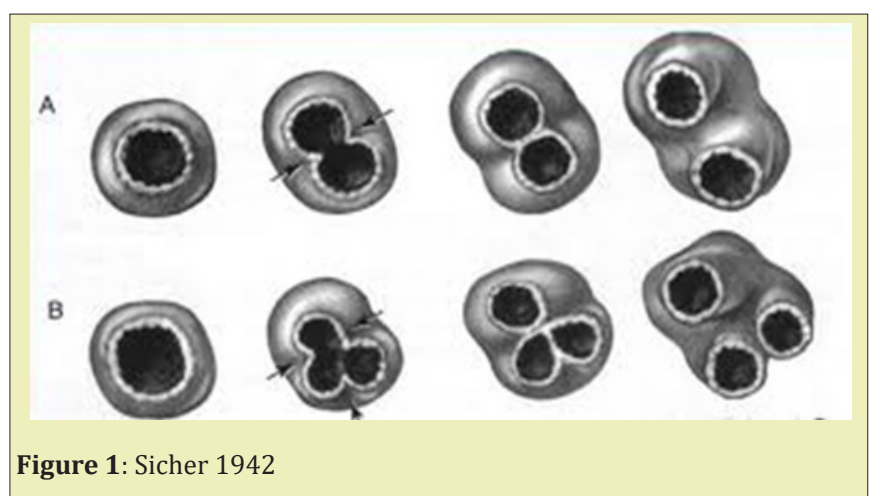

Figure 1: Sicher 1942

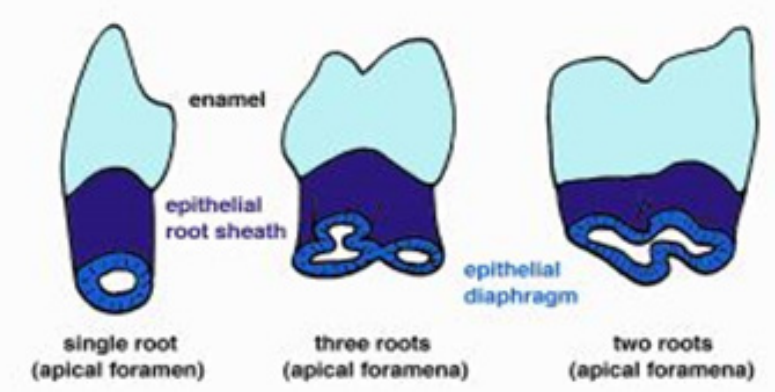

Figure 2: Anterior teeth.

The formation of the floor of the pulp chamber has received little information up to now. Inter-radicular dentinal processes give rise to the floor of the pulp chamber. Two or three inter-radicular dentinal processes give rise to the floor of the pulp chamber. The crista interradicularis leads to the formation of a bridge further mineralized. Failure of fusion leads to the formation of perio-periodental surnumerary canals and constitute another difficulty during endodontic treatment, due to the presence of fistula.

\section{Cement formation}

Cement formation occurs along the entire tooth. It is an extension of the inner and outer dental epithelium. Ecto-mesenchymal cells are issued from the inner portion of the dental follicle. They differentiate into cementoblasts. In the upper part of the root the cementum is a cellular, whereas in the lower portion, cellular cementum is formed. ${ }^{5}$

\section{Tooth eruption}

The apical part of the root stays constantly at the same location (in the epithelial hammock) whereas the lengthening of the root leads to tooth eruption in the mouth (oral cavity) Figure 3. Tooth eruption occurs after initiation of the root formation along the epithelial diaphragm. The apical part of the tooth is formed by three distinct layers Figure 4 \& Figure 5.

Stem Cells of the Apical Papilla (SCAP) are the source of primary odontoblasts that synthesize primary dentin. They are involved in the root formation and lengthening. In the inner part of the root, in the pulp, radicular dental pulp, the apical papilla mesenchyme and the apical cell-rich zone are firstly transported from the apex to a more central zone. Afterward, they migrate toward a sub-odontoblastic border (the so-called Höhl zone), and later display lateral sliding from the apical mesenchyme toward the radicular dental pulp. The SCAP cell migration moves from the root toward the crown, where they acquire a terminal phenotype. Along the outer part of the root, different cementum layers are found: namely Acellular Afibrillar Cementum (the half of the upper part of the root) followed by Cellular Cementum (lower part of the root) Figure 6. In the molars of adult mice, acellular cementum is formed in the upper part, whereas cellular cementum is formed in the lower part of the root $^{6}$ Figure 7.

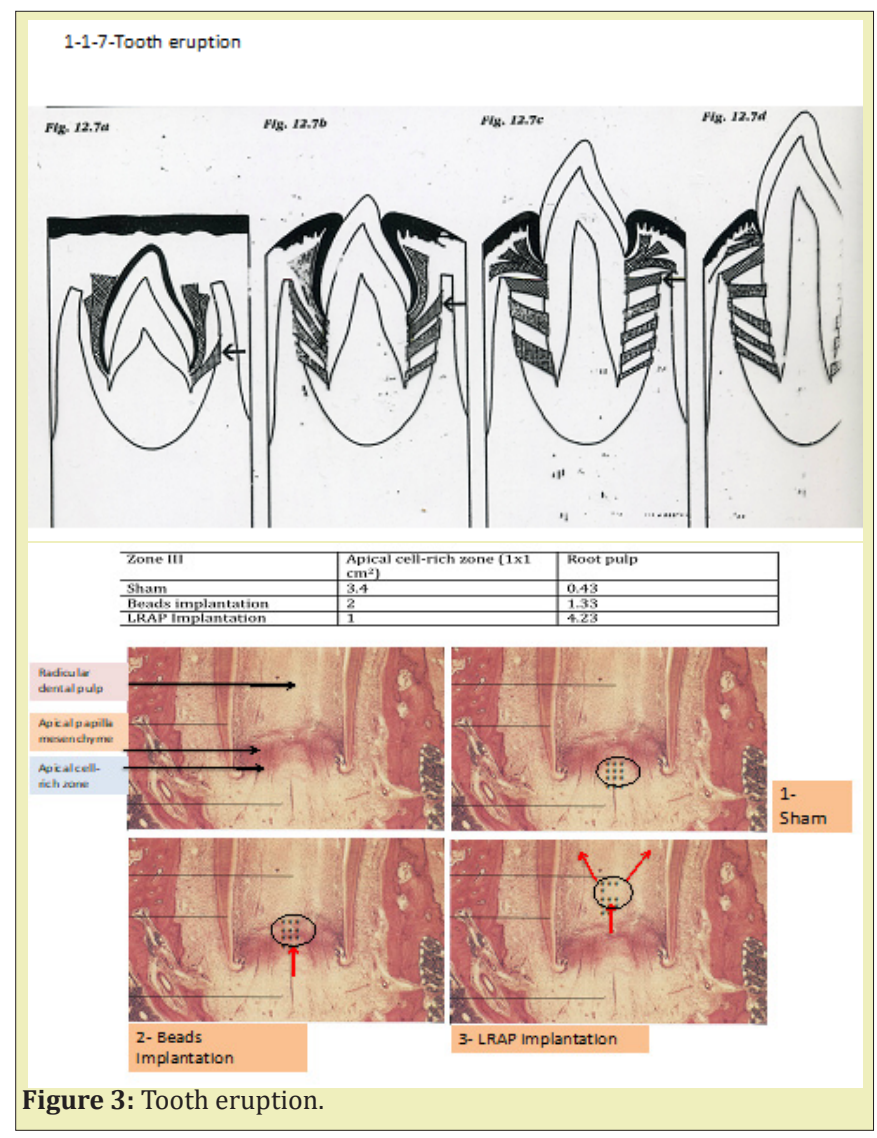

\section{Epithelial diaphragm}

The IEE and OEE join at the neck of the forming tooth. This is called the zone de reflexion or cervical loop. Fibronectin was distributed at the interface between the epithelial diaphragm and pre-odontoblasts. Type I collagen was absent, as it was the case between the epithelial diaphragm and pre-odontoblasts. In contrast, type III collagen showed strong staining at this location. The epithelial diaphragm was presumed to be involved in tooth eruption Figure 8. ${ }^{7}$ Root formation and epithelial cells rest of Malassez. Ater dissociation of the Hertwig's Epithelial Root Sheath, epithelial cell rest of Malassez are part of the periodontal ligament and they are located inside the cellular cementum. 

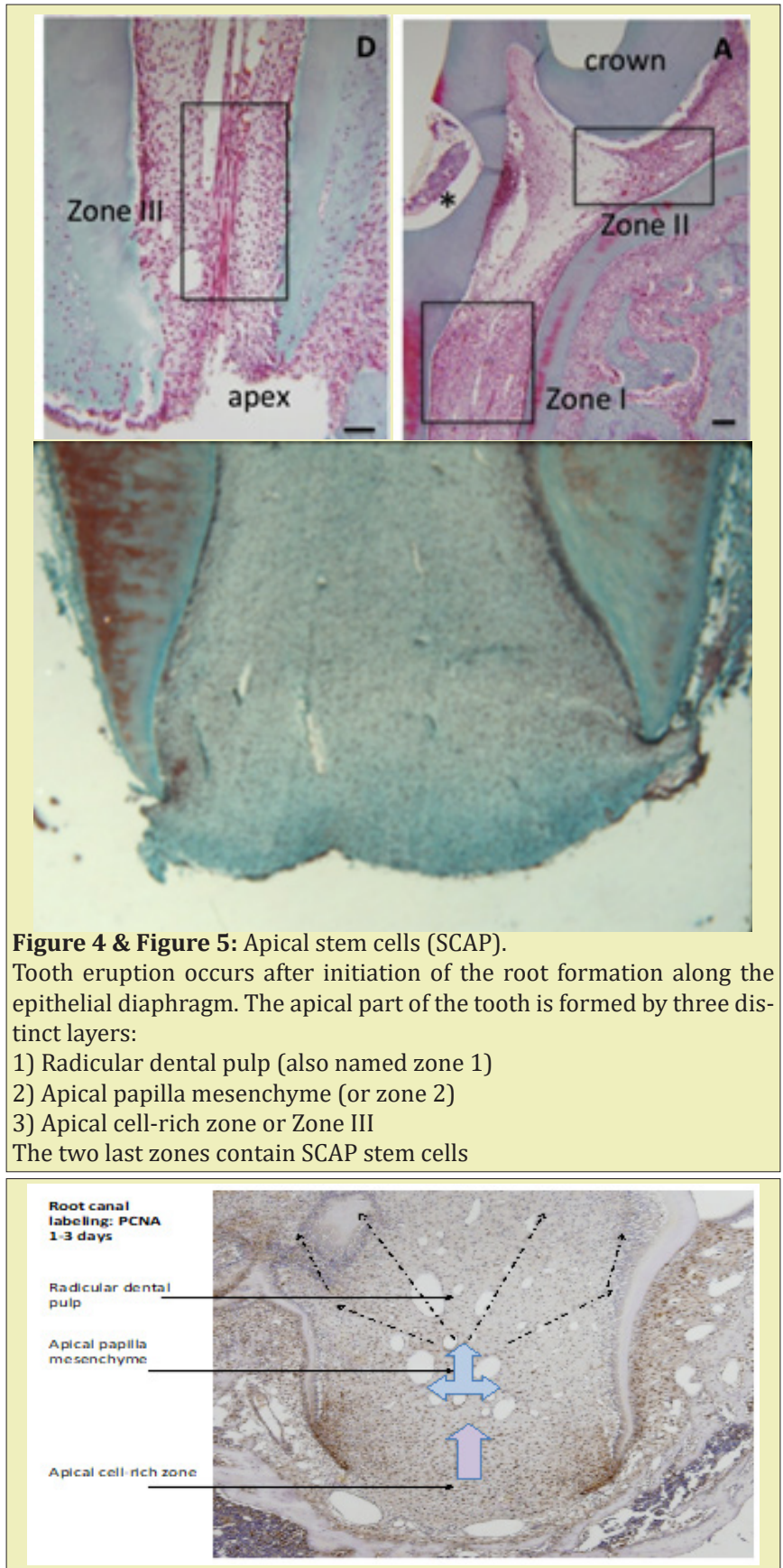

Figure 6: Cell sliding in the apical part of the tooth.

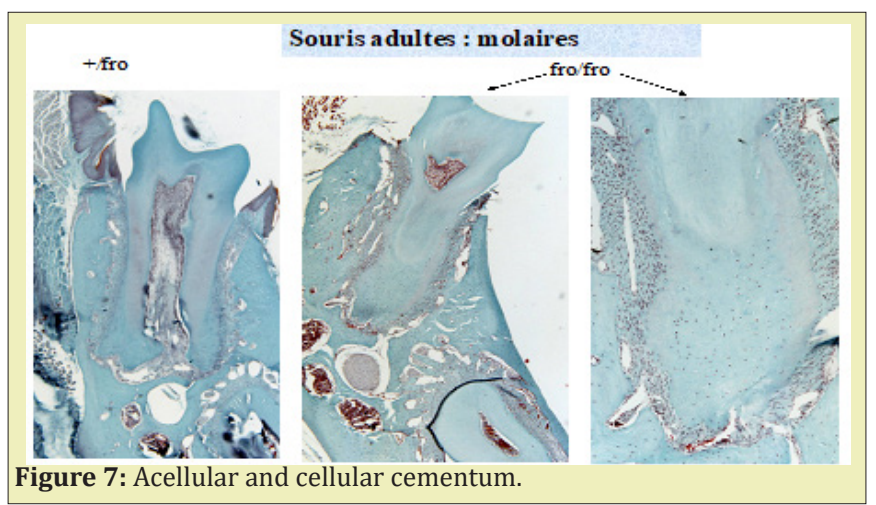

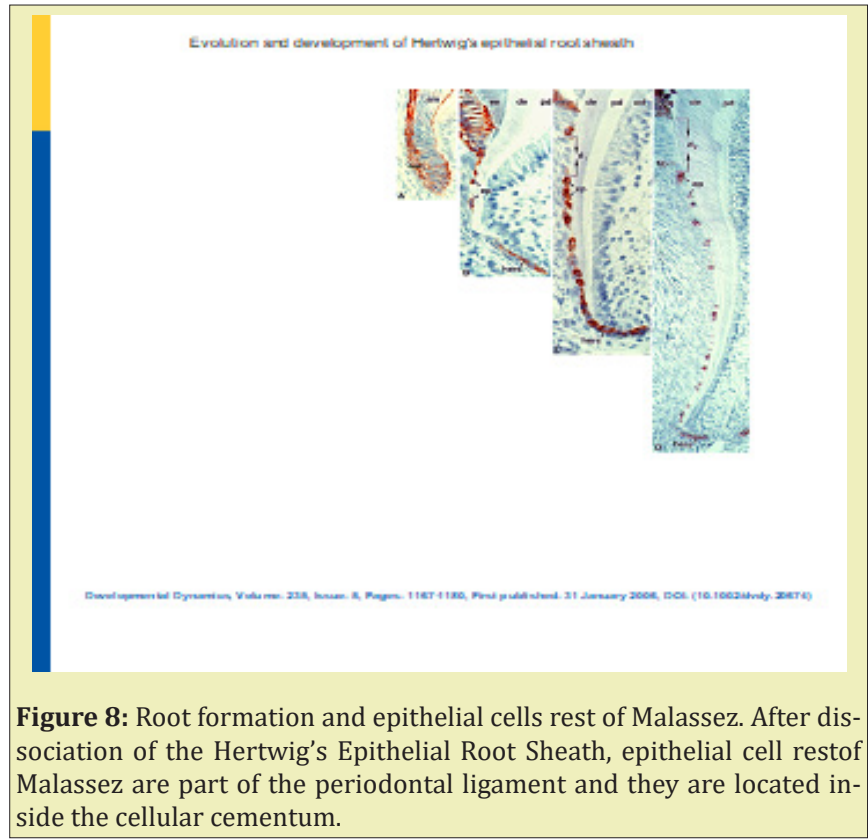

\section{Apexogenesis/Apexification}

\section{Root formation (apexogenesis)}

Primary dentin is formed until the full length of the root development is reached. This is followed by dentin formation that proceeds as secondary dentinogenesis and the associated tooth eruption. The outer and inner enamel epithelia constitute the two layers of the Hertwig's sheath, derived from the inner and outer enamel epithelia. They fuse below the level of the cervical margin of the crown. These layers are responsible for root formation. ${ }^{3}$ The cells of the Hertwig's epithelial root sheath ${ }^{2}$ are linked by desmosomes, hemi-desmosomes and gap junctions. They are adherent to the inner and outer basement membranes. Mesenchymal cells of the dental follicle become cementoblasts. Cells from the dental sac cross the dissociated HERS. They migrate through the dissociations and become cementoblastes and later cementocytes. The outer layer disintegrates. Between the dissociated epithelial outer layer, the cells invade the surface of root dentin (forming the so-called mantle dentin). In contrast, a cellular cementum and cementogenesis take origin from the inner epithelial cells. Apexogenesis continues at a slower rate throughout the lifetime of the individual. As the root and pulp develop, the dental papilla located apically to the developing pulp contributes to the root formation. HERS is very sensitive to trauma and once destroyed by trauma or by infection, the normal root development is stopped without further differentiation of odontoblasts. $^{8}$

\section{Formation of the apical zone}

A series of dental tissues are formed in the apical zone. They include 1) the apical cell-rich zone, 2) the apical papilla mesenchyme and 3) the radicular dental pulp. Apexogenesis involves both 
lengthening (associated with eruption of the teeth), and the thickening of the dentin layer, to the detriment of the lumen (pulp space). Apexogenesis result from the contribution of cells issued from the dental papilla. They become after differentiation, odontoblasts, involved in the production of dentin, whereas differentiation of cells from the cervical loop contribute to the lengthening of the tooth. Apexogenesis is a natural physiologic process of root development. The term is used to describe the preservation of pulp vitality. It has also been suggested that maturogenesis is a more appropriate term than apexification, because not only the apex but the entire root is allowed to mature. ${ }^{9}$

Calcium hydroxide induced the deposition of calcified material. It became the standard treatment protocol for the therapy of non-vital immature tooth. Many other biomaterials have been used to induce apexification. Calcium hydroxyde-induced apexification might require 6-24 months for barrier formation. The barrier formed is often porous and not continuous. Further development of the root does not take place. The rationale of revascularization is that if a sterile tissue matrix is provided in which new cells can grow, pulp vitality can be re-established. It provides a matrix into which the cells from the periapical tissues could grow and re-establish pulp vascularity, slowly replacing the necrotic tissue.

It is possible that a few vital pulp cells remain alife at the apical end of the root canal. These cells may proliferate into the newly formed matrix and differentiate into odontoblasts. The newly formed odontoblasts can lay down atubular dentin at the apical end, causing apexogenesis, as well as on lateral aspects of dentinal walls of the root canal, reinforcing and strengthening the root. ${ }^{10}$

A. The blood clot is a reservoir of growth factors. The advantage is that achieving continued root lengthening and strengthening, the result is a reinforcement of lateral dentinal walls with deposition of new dentin/hard tissue. Complete root development requires a viable pulp containing cells that can differentiate into dentin-producing odontoblasts. The dental pulp is complex with a variety of cells, nerves, and blood vessels. It is important to keep in mind the required prerequisites, including: 1) cells that are capable of differentiating into pulp cells synthesizing signals that are required for the cell differentiation, 2) inside an appropriate scaffold, suitable for guiding regeneration of the tissues. The procedure that induces apexogenesis is undertaken to preserve the remaining vital tissue and allow completion of root formation and apical maturation. 3) Apexification is related to the immature teeth.

B. Apexification is then performed to treat immature teeth with non-vital pulp by inducing a calcified barrier at the open apex.

C. Apexogenesis is a natural physiologic process of root development. However, the term is used commonly to describe the endodontic procedure of preservation of pulp vitality in a traumatized tooth with pulp involvement, so that the affected tooth could develop its full growth potential. ${ }^{11}$

D. It was earlier unthinkable that the tissue in the periapical region of a nonvital infected tooth growth factor, vascular endothelial growth factor (VEGF), platelet-derived epithelial growth factor, and other tissue growth factors which could stimulate differentiation, growth, and maturation of fibroblasts, odontoblasts, and cementoblasts. It is possible that a few vital pulp cells remain at the apical end of the root canal. These cells might proliferate into the newly formed matrix and differentiate into odontoblasts under the organizing influence of cells of Hertwig's epithelial root sheath, which are quite resistant to destruction, even in presence of inflammation processes.

E. Another mechanism may be attributed to the stem cells located in the periodontal ligament, which can proliferate, grow into the apical end and within the root. They may deposit hard tissue both at the apical end and on the lateral root walls.

F. A third possibility could be attributed to the stem cells from the apical papilla or the bone marrow.

Instrumentation beyond the confine of the root canal is susceptible to induce bleeding. This may also transplant mesenchymal stem cells from the bone into the canal lumen. These cells have extensive proliferating capacity. The blood clot itself, being a rich source of growth factors, could play an important role in regeneration.

\section{Apexification}

Pulp necrosis arrest further root development of an immature permanent tooth. To induce the formation of an apical mineralized tissue barrier, the method used specifically is named apexification because of its high $\mathrm{pH}$, calcium hydroxide not only weakens the root but may also inhibit new tissue formation within the canal. The possibility of vital tissue regeneration in the root canal space with a continuous increase in root thickness and length has been demonstrated for immature teeth. The apical part of the root includes three compartments: 1) an apical cell-rich zone (where apical stem cells are mostly located and constitute a reservoir of undifferentiated pulp cells progenitors SCAP cells,2) cells located in the apical papilla mesenchyme, and 3) in the radicular dental pulp.

There are three major components in tissue engineering which are implicated in apexification: Cells that are capable of hard tissue formation (differentiated odontoblasts), Scaffolds that can support cell growth and differentiation, Molecules that provide signaling and intracanalar dentin formation.

Dental pulp stem cells are able to differentiate into functional odontoblast-like cells with an active mineralization potential. 
They may be used in dental tissue engineering via stem cell-based approaches. This is due to an increased alkaline phosphatase activity, dentine sialoprotein expression and to the formation of mineralized nodules. Platelet-rich plasma is a natural reservoir of various growth factors that can be collected, unlike the chemically processed molecules or recombinant proteins that may cause undesired side effects and expose the tissue to unnecessary risks. The use of platelet-rich plasma (PRP) in combination of DPCs may be beneficial for new tissue formation and for apical closure. The protocol for pulp revascularization/revitalization begins with root canal irrigation with minimal instrumentation and then continues with disinfection with an antibiotic mixture. The most commonly reported dressing is a triple antibiotic paste (TAP), which consists of ciprofloxacin, metronidazole, and minocycline. It is presumed that the blood clot serves as a scaffold in which stem cells from the apical papilla (SCAP) populate the clot. In addition, growth factors released from platelets and the dentinal walls serve as a promoter for stem cell division and differentiation processes.

\section{Immunohistochemistry}

Gene profile analysis have identified perivascular cells by using markers, such as alkaline phosphatase and $\alpha$-smooth muscle actin, in differing proportions on STRO-1 positive cells. BMPs appear to be the key regulators of apexification. The cell lines are grown and expanded before being implanted into the root canal, resulting in protracted clinical treatment times. The implanted cells need to reliably adhere to the disinfected root canal walls. The implanted tissue lacks a crucial vascular supply, and it is technically difficult to replant the three-dimensional regenerated pulp without damaging the cells. When an open root apex exists, a similar scaffold design adjacent to a vascular supply may assist apexification by thickening and closing the apical portion of the root with hard tissue. Novel genes and appropriate vectors are mandatory to control cell-specific safe delivery. The phenotype repopulating the open root apex has still to be selected by environmental factors.

\section{The goals of apexogenesis are the followings:}

a) Allowing a continued development of root length for a more favorable crown-to-root ratio.

b) Allowing the remaining odontoblasts to lay down dentin, producing a thicker root and decreasing the risks of root fracture.

c) Promoting root end closure, creating a natural apical constriction for root canal filling.

d) Generating a dentinal bridge at the site of pulpotomy. It suggests that the pulp has maintained its vitality. Then laceration of the periapical tissues cause bleeding into the root canals. If vital and not irreversibly inflammed, maintenance of pulpvitality will allow natural continued root developmentby using apexogenesis. This allow continued root development along the entire root length. Depending on the extent of inflammation, pulp capping, or conventional pulpotomy may be indicated. Traditionally, the approach has been to use calcium hydroxide $(\mathrm{CH})$ to induce apexification after disinfection of the root canals.

Laceration of the periapical tissues cause bleeding into the root canals. If vital and not irreversibly inflamed, maintenance of vitality will allow natural continued root development. Maintenance of pulp vitality by using apexogenesis allows continued root development along the entire root length. Depending on the extent of inflammation, pulp capping, or conventional pulpotomy may be indicated. It is important to note that apexification by either $\mathrm{Ca}(\mathrm{OH})_{2}$ or MTA completely prevents any further root development in terms of increased radiographic measures of either root length or width. The immature tooth treated by apexification procedures demonstrates healing of apical periodontitis, but does not achieve the goals of continued root development or restoration of functional pulp tissue.

Apexification is defined as 'a method to induce a calcified barrier in a root with an open apex or the continued apical development of an incomplete root in teeth with necrotic pulp'. Success rates for calcium hydroxide apexification is a method of inducing apical closure through the formation of mineralized tissue in the apical pulp region of a non-vital tooth with an incompletely formed root. It is composed of osteocementum, osteodentin or bone, or by some combination of the three.

Two new clinical concepts have emerged recently. One involves a revitalization approach to achieve tissue generation and/or regeneration. In this method, new living tissue is expected to form in the cleaned canal space, allowing continued root development in terms of both length and thickness. The other is the active pursuit of pulp/dentine regeneration via tissue engineering technology to implant or re-grow pulps.

The other new protocol has the potential to benefit immature pulpless teeth by allowing continued growth and maturation. A haemorrhage is induced to fill the canal with blood clot as a scaffold to allow generation of live tissues in the canal space and continued root formation (length and wall thickness). Attempts to regenerate pulp tissue have been considered impossible until recently and major developments in two basic researches, namely tissue engineering and stem cell biology Figure $9 \&$ Figure 10.

\section{Apexification}

i. Figure 11 Different apical closure types in group dental pulp cells + platelet rich plasma. Some bone like tissue form a bridge and merge with cementum-like tissue to close the apex. Reprinted from Nosrat. ${ }^{12}$ 


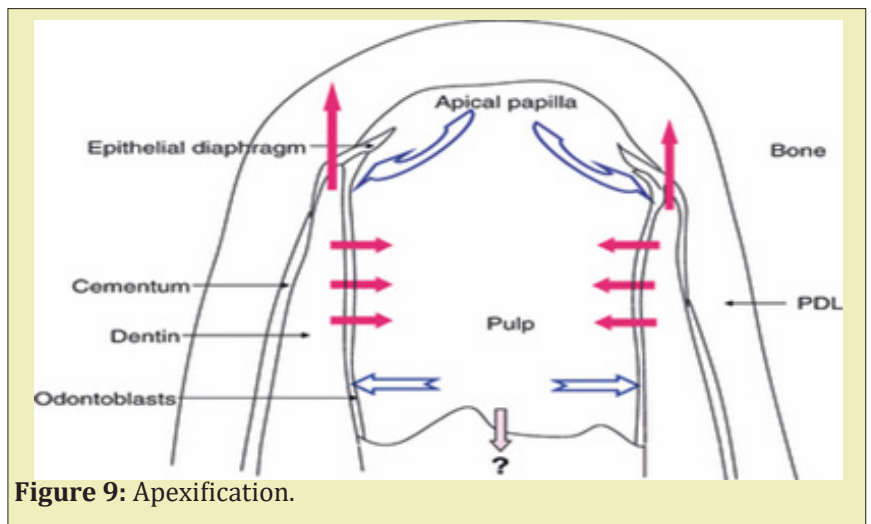

Figure 9: Apexification.

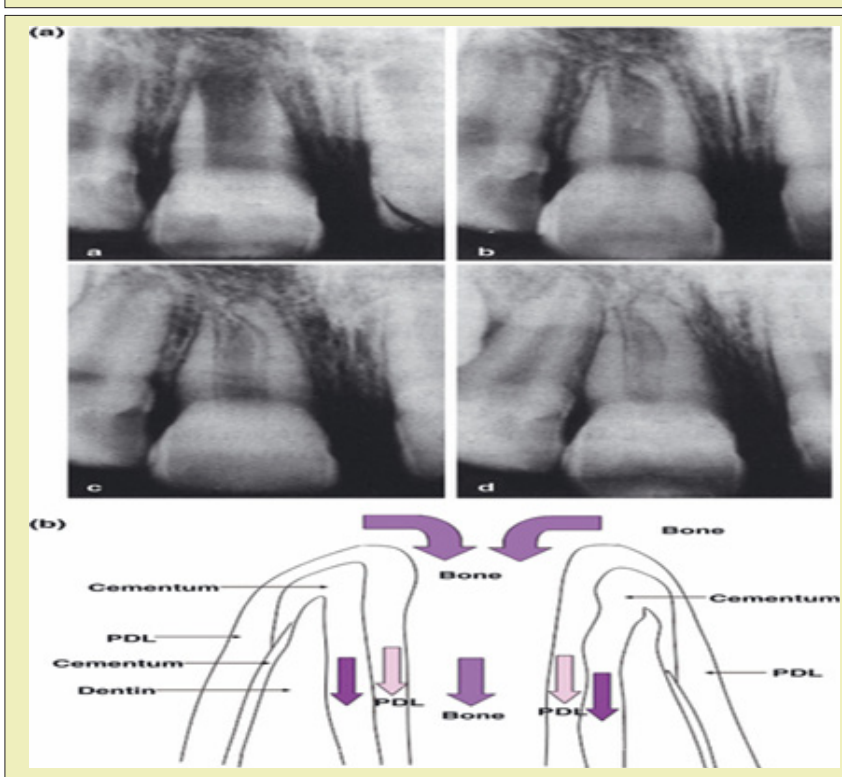

Figure 10: Apexification.

ii. Apexification is a method of inducing a calcified apical barrier or continued apical development of an incompletely formed root in which the pulp is necrotic. The developing consensus approach to accomplish apexification is to instrument root canals, to remove the necrotic tissue, and to place MTA in the root canal apex, with the remainder of the canal obturated with gutta-percha Figure 11.

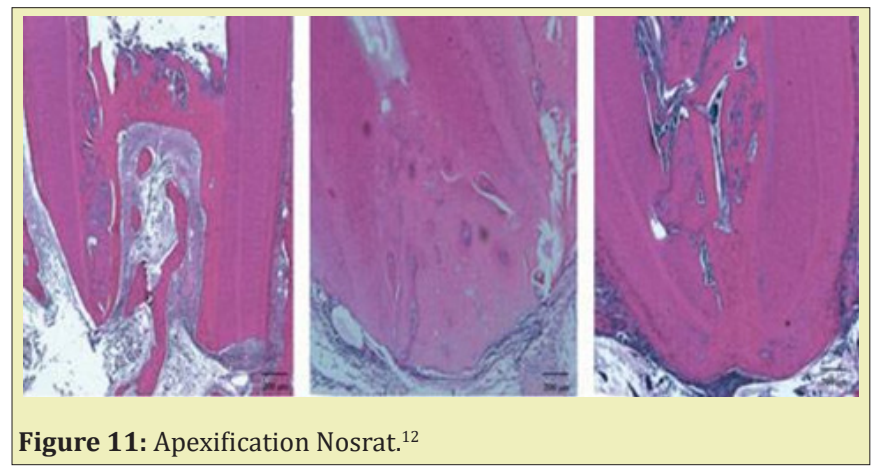

iii. Apexification. In the absence of a vital pulp, dentin deposition is arrested. When an immature tooth is affected by caries or trauma, the pulp requires proper management according to the degree of inflammation and keeping some vitality. Artificial apical barriers are formed after implantation of a variety of materials. Apexification was demonstrated in a complete layer of cementum when using MTA as a root-end filling inducing apical hard tissue formation in immature roots. An alternative treatment of the immature permanent tooth is apexification procedures. The classic apexification method involves long term application of $\mathrm{Ca}(\mathrm{OH})_{2}$. A more recent method of apexification involves the use of MTA as an apical barrier followed by placing either a root filling or obturating material. However, it is important to note that apexification by either $\mathrm{Ca}(\mathrm{OH})_{2}$ or MTA completely prevents any further root development in terms of increased radiographic measures of either root length or width. The immature tooth treated by apexification procedures demonstrates healing of apical periodontitis, but does not achieve the goals of continued root development or restoration of functional pulp tissue.

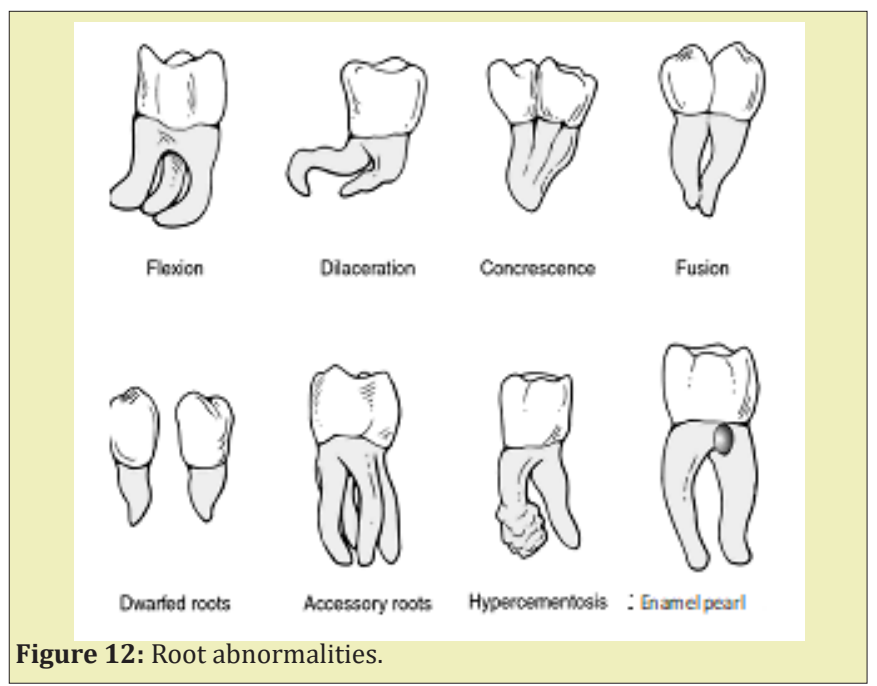

Figure 12: Root abnormalities.

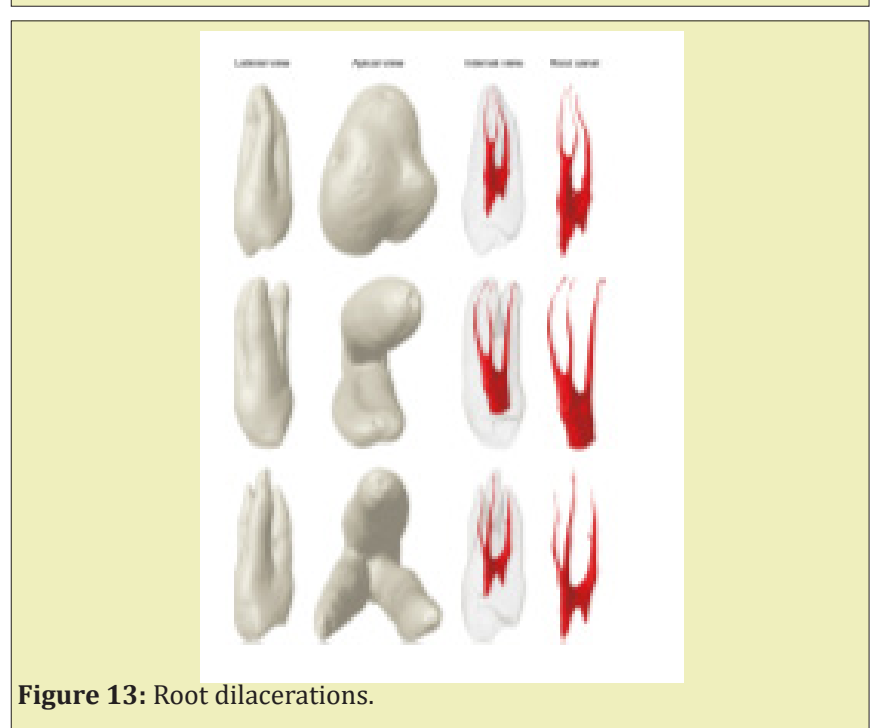

iv. Apexification is defined as 'a method to induce a calcified barrier in a root with an open apex or the continued apical devel- 
opment of an incomplete root in teeth with necrotic pulp'.

v. Success rates for calcium hydroxide apexification are high purification is a method of inducing apical closure through the formation of mineralized tissue in the apical pulp region of a non-vital tooth with an incompletely formed root. It is composed of osteocementum, osteodentin or bone, or by some combination of the three.

vi. Apexification may be induced by $\mathrm{Ca}(\mathrm{OH})_{2}$ or MTA. Revascularization of necrotic pulp has been considered possible after traumatic injury to an immature tooth. A unique set of circumstances exist that favor revascularization. The potential for revascularization appears to directly depends on the race between bacterial infection of the necrotic pulp, and revascularization of the canal space using the ischemic pulp as a matrix. Revascularization involves desinfection the root canal, providing a blood clot into which cells could grow and seal the coronal access. The canals were sampled before and after irrigation with $1.25 \%$ NaOCL and after dressing with a triple antibiotic paste, consisting of metronidazole, ciprofloxacin, and minocycline. The access cavity was sealed with glass ionomer cement (GIC). An increased tooth length was observed. Revascularization procedures may be conducted on immature non-vital, infected permanent teeth. An endodontic regeneration study on non-human primates by cleaning and shaping root canals.

vii. Metronidazole is a nitroimidazole compound that exhibits a broad spectrum of activity against protozoa and anaerobic bacteria. It has been used both systemically and topically in the treatment of periodontal disease. Metronidazole readily permeates bacterial cell membranes. It binds to the DNA, disrupting its helical structure, and leads to rapid cell death. Tetracyclines, which include doxycycline and minocycline, are effective against most spirochetes, and many anaerobic and facultative bacteria. The tetracyclines gain access to bacterial cells. They act by inhibiting protein synthesis on the surfaces of ribosomes. Minocycline is a semi-synthetic derivative of tetracycline.

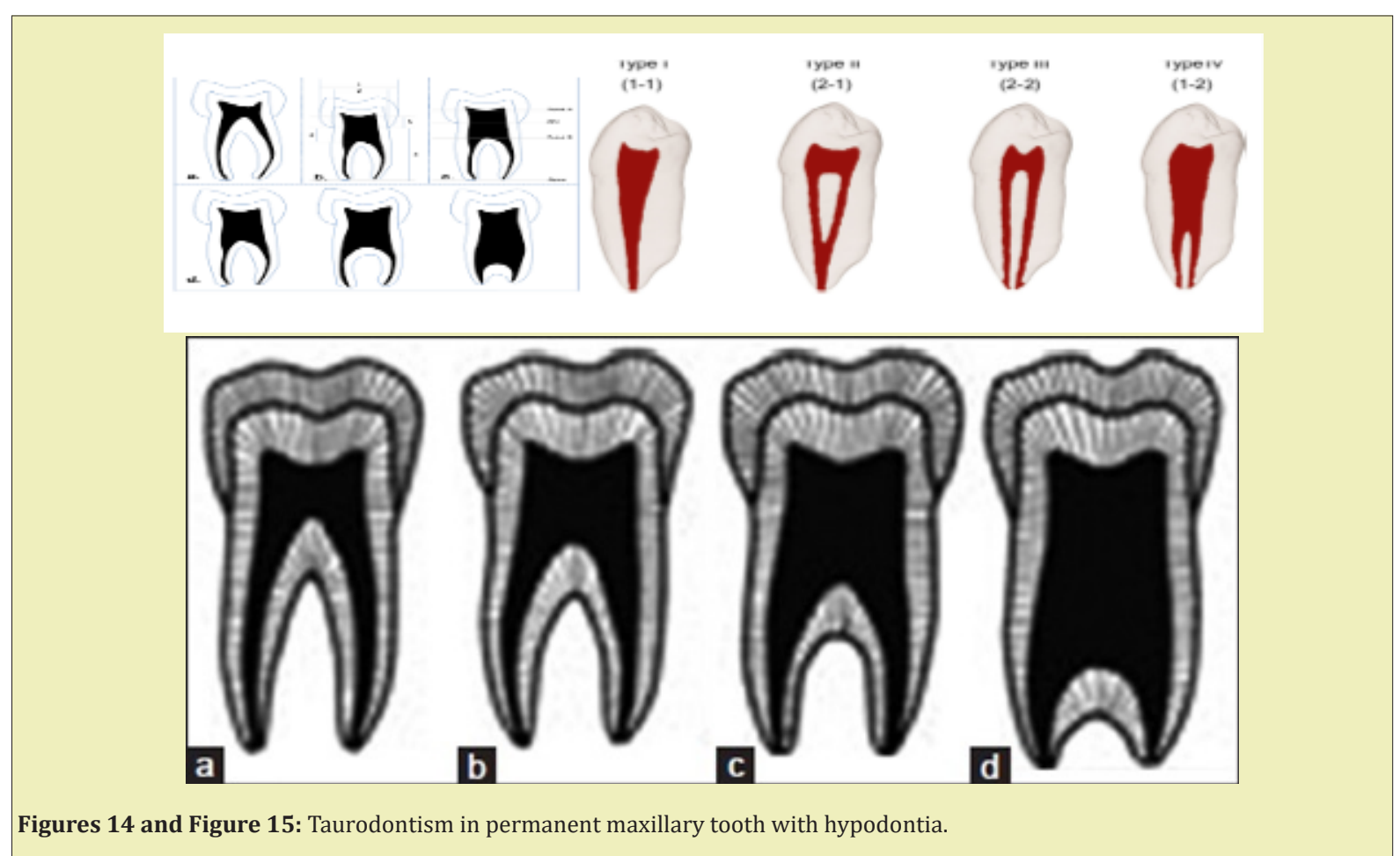

viii. It is available in many topical forms ranging from gel mixtures to sustained release microspheres. Ciprofloxacin has a bactericidal mode of action and very potent activity against gram-negative pathogens but displays limited activity against gram-positive bacteria. Most anaerobic bacteria are resistant to ciprofloxacin. Side effects of ciprofloxacin have been reported. It was found that the drug is clinically safe when applied in low doses. When applied as an intra-canal medicament in low doses, adverse systemic side effects should be minimized. ix. In addition to direct or indirect capping stimulating reparative dentin formation, apexogenesis is the most usual tool for the treatment of endodontic lesions, namely when the pulp is still alife. Apexification constitutes the most viable endodontic method for a dental pulp either necrotic, infected or not. After disinfection using a triple-antibiotic therapy, the radicular pulp may regenerate, become functional and restore most of its activities. 


\section{Classification of Root Abnormalities}

Roots malformations may be subdivided into 1) disorders of root development alone, and 2) disorders of the roots associated with a general tooth dysplasia:

\section{Genetic defects disorders of root development include developmental abnormalities}

Hertwig's epithelial root sheath (HERS)influence root formation and the formation of furcation in multi-rooted tooth.

Hypophosphatasia disrupt the formation of acellular cementum. Blood phosphate controls the cementum formation and inhibits mineralization.

Teeth may be missing (hypodontia), or in excess (supernumary teeth or hyperdontia) or mis-shapped.

a. Transition from crown to root development

b. Apical growth of HERS associated with root elongation

c. Induction of odontoblast differentiation and radicular dentinogenesis

d. Disintegration of HERS and initiation of cementogenesis

e. Formation of acellular and cellular cementum

\section{Premature arrest of root formation}

Apical infection, radiation, chemotherapy, influencing Nfic, Ptc, Dkk1, Osx, Smad4 and Wls genes.

\section{Root dilacerations}

Sharp bend of the crown or root axis, with abnormal curvature of the root. It is the result of a developmental anomaly in which there has been an abrupt change in the axial inclination between the crown and the root of a tooth, but the criteria in the literature for recognizing root dilaceration vary. Two possible causes of dilaceration are trauma and developmental disturbances, and it has also been proposed that it might be associated with some developmental syndromes. Dilaceration can be seen in both the permanent and deciduous dentitions, and it is more commonly found in the maxilla posterior teeth. Periapical radiographs are the most appropriate way to diagnose the presence of root dilacerations. Diagnosis, endodontic access, cavity preparation, root canal preparation and filling, and other related treatments might be complicated by the presence of a dilacerations Luder. ${ }^{9}$ Dilaceration results of a developmental anomaly in which there has been an abrupt change in the axial inclination between the crown and the root, but the criteria in the literature for recognizing root dilaceration vary. Dilaceration can be seen in both the permanent and deciduous dentitions, and it is more commonly found in the maxilla posterior teeth. Periapical radiographs are the most appropriate way to diagnose the presence of root dilacerations. Diagnosis, endodontic access, cavity preparation, root canal preparation and filling, and other related treatments might be complicated by the presence of a dilacerations.

Root malformation associated with a cervical mineralized diaphragm/molar incisor malformation.

Short root anomaly Trisomy 21 is frequently associated with short roots.

Long roots are associated with oculo-facio-cardio-dental (OFCD) syndrome.

Taurodontism: Apical displacement of the bi-or trifurcation. An increased occluso-apical height of the pulp cavity is observed. Taurodontism is prevalent in Down's syndrome. Disorders of root development associated with general tooth dysplasia associated with a general tooth dysplasia include:

Double teeth union of two adjacent teeth during odontogenesis. The number of teeth is normal in case of germination and reduced in case of fusion. Extra multi-roots are seen in mandibular premolars.

Hypophosphatasia disrupt the formation of acellular cementum. There is an Inhibition of mineralization in the furcation in multi rooted tooth.

a. The transition from crown to root development may be altered

b. Apical growth of HERS may be associated with root elongation

c. Induction of odontoblast differentiation and radicular dentinogenesis

d. Disintegration of HERS and initiation of cementogenesis

e. Formation of acellular and cellular cementum Figure 12-Figure 15.

\section{Regional Dysplasia}

Dentin dysplasia type I. Dentinogenesis Imperfecta Types I, II, and III, and X-linked hypophosphatemia. Different types of DI are caused by the genetic defects of COLIA1 and COLIA2, the two genes coding for the $\alpha 1$ and $\alpha 2$ chains of type I collagen. DI exhibits extensively large pulp cavities

Genetic defects: alterations of Nfic, Ptc, Dkk1, Osx, Smad4, and Wls have been identified in human teeth. In man, two main forms exist: the CLCN7 encoding a chloride channel, and the second is related to a defective PLG gene (encoding plasminogen).

\section{Conclusion}

The Inner and Outer layers of the Hertwig's Root Sheath contribute to root lengthening and dentin thickening. Apexification of the dental root is a crucial event implicated in root formation. In 
addition to technical difficulties, apical closure contributes to the healing of periapical lesions. Cementogenesis, acellular and cellular cementum are also implicated in root formation. Defective root structures add to endodontic difficulties. Dilacerations, taurodontism, short and long root anomalies, mis-shaped roots structures interfere with genes regulating root formation, such as Nfic, Ptc, Dkk1, Osx, Smad4 and Wls. Alteration of a chloride channel and/or defective plasminogen gene are linked to local alterations, also involved in regional dysplasia (such as Type I dentin dysplasia).

\section{Acknowledgments}

None.

\section{Funding}

None.

\section{Conflicts of Interest}

Author declares that there is no conflict of interest.

\section{References}

1. Hirata A, Nakamura M. Localization of perlecan and heparanase in Hertwig's epithelial root sheath during root formation in mouse molars. J Histochem Cytochem. 2006;54(10):1105-1113.
2. Fong CD, Hammarstrom L. Expression of amelin and amelogenin in epithelial root sheath remnants of fully formed rat molars. Oral Surgery, oral medicine, oral pathology, oral radiology. 2000;90(2):218-223.

3. Thomas HF. Root formation. Int J Dev Biol. 1995;39:231-237.

4. Sicher H. The axial movement of continuously growing teeth. J Dent Res. 1942;21(2);201-210.

5. Ooe T. Human tooth and dental arch development. Ishiyaku Publishers, Inc. Tokyo, Osaka. 1981.

6. Vertucci FJ. Root canal morphology and its relationship to endodontic procedures. Endodontic Topics 2005;10(1):3-29.

7. Huang GTJ. Apexification: the beginning of the end. Int Endod J. 2009;42(10):855-866.

8. Ahmed HMA, Versiani MA. A new system for classifying root and canal morphology. Int Endod J. 2017;50(8):761-770.

9. Luder HU. Malformations of the tooth root in humans. Front Physiol. $2015 ; 6: 307$

10. AhmedHMA, Hashem AAR, Dummer PMH. Application of a new system for classifying root and canal anatomy in clinical practice-Explanation and elaboration. Eur Endo J. 2021;6(2);132-142.

11. Ahmed HMA, Dummer PMH. A new system for classifying tooth, root and canal anomalies. International Endod J. 2018;51:389-404.

12. Nosrat A, Li KI, Vir K, et al. Is pulp necessary for root maturation. J Endod. 2013;39(10):1291-1295. 